\title{
Development, performance and application of novel GaN-based micro-LED arrays with individually addressable n-electrodes
}

Enyuan Xie, ${ }^{1}$ Mark Stonehouse, ${ }^{1}$ Ricardo Ferreira, ${ }^{1}$ Jonathan J. D. McKendry, ${ }^{1}$ Johannes Herrnsdorf, ${ }^{1}$ Xiangyu He, ${ }^{1}$ Sujan Rajbhandari, ${ }^{2}$ Hyunchae Chun, ${ }^{2}$ Aravind V. N. Jalajakumari, ${ }^{3}$ Oscar Almer, ${ }^{3}$ Stefan Videv, ${ }^{4}$ Grahame Faulkner, ${ }^{2}$ Ian M. Watson, ${ }^{1}$ Erdan Gu, ${ }^{1}$ Robert Henderson, ${ }^{3}$ Dominic O'Brien, ${ }^{2}$ Harald Haas, ${ }^{4}$ Martin D. Dawson ${ }^{1}$

${ }^{1}$ Institute of Photonics, Department of Physics, University of Strathclyde, Glasgow, G1 1RD, UK

${ }^{2}$ Department of Engineering Science, University of Oxford, Oxford, OX1 3PJ, UK

${ }^{3}$ CMOS Sensors and Systems Group, University of Edinburgh, Edinburgh, EH9 $3 \mathrm{JL}, \mathrm{UK}$

${ }^{4} L i-F i R \& D$ Centre, the University of Edinburgh, Institute for Digital Communications, King's Buildings, Edinburgh, EH9 3JL, UK.

Abstract - We demonstrate the development, performance and application of a GaN-based micro-light emitting diode array sharing a common p-electrode with individual-addressed n-electrodes. These individually-addressed $n$ electrodes minimize the series-resistance difference from conductive paths, and offer compatibility with n-type metal-oxide-semiconductor transistor-based drivers for faster modulation.

\section{INTRODUCTION}

GaN-based micro-light-emitting diode ( $\mu$ LED) arrays, which consist of a number of $\mu$ LED elements with dimensions of less than $100 \mu \mathrm{m}$, possess important novel characteristics. Compared with conventional broadarea LEDs, $\mu$ LED elements can be operated at higher current densities, enabling significantly higher modulation bandwidth for data communications applications [1]. By operating a $\mu$ LED array in a ganged fashion (multiple $\mu$ LED elements modulated simultaneously with the same data signal), a higher signal-to-noise ratio and longer data-transmission distance are expected while retaining fast data rate [2]. This makes $\mu$ LED arrays attractive sources for high-speed visible light communications (VLC) in both polymer waveguide and free-space formats.

Standard GaN-based LED epitaxial structures have the p-side of the junction on top. This epi-structure requires that a conventional $\mu$ LED array employs a configuration with a common n-electrode and individually addressable p-electrodes for each $\mu$ LED element. The main shortcoming of this configuration is that the relevant LED drivers are necessarily based on p-type metal-oxide-semiconductor (PMOS) transistors, which have lower operation speed, larger size and larger capacitance than their n-type equivalents. In addition, this configuration employs the n-type GaN layer as a shared conductive path for all $\mu$ LED elements. Different distances between the common n-electrode and the target $\mu$ LED element lead to different series resistances contributed from the $\mathrm{n}$ type GaN layer, which results in poor optical element-to-element uniformity and high crosstalk.

In this work, we demonstrate a novel GaN-based $\mu$ LED array sharing a common p-electrode with individually addressable n-electrodes. Compared with a conventional $\mu$ LED array, the reversed common and individual electrode structure of this configuration minimises the series-resistance difference from conductive paths, and offers compatibility with NMOS-based LED drivers. We have developed and optimised the fabrication process for such arrays to improve performance. At $10.5 \mathrm{kA} / \mathrm{cm}^{2}$ operating current density, over $414 \mathrm{~W} / \mathrm{cm}^{2}$ optical power density and $345 \mathrm{MHz}$ electrical-to-optical (E-O) modulation bandwidth are achieved for a single $\mu$ LED element with a diameter of $24 \mu \mathrm{m}$ emitting at $450 \mathrm{~nm}$. This array was also integrated with a custom NMOS-based driver to demonstrate VLC application. Open eye diagrams were recorded at several hundred Mbps in operation with two $\mu$ LED elements under an on-off-keying (OOK) data transmission scheme.

\section{FABRICATION PROCESS FOR $\mu$ LED ARRAYS}

The $\mu$ LED arrays developed in this work were fabricated from a commercial blue GaN-based LED wafer on a cplane sapphire substrate. The $\mu \mathrm{LED}$ array consists of $6 \times 6$ array of flip-chip $\mu \mathrm{LED}$ elements with a diameter of $24 \mu \mathrm{m}$ on a $300 \mu \mathrm{m}$ centre-to-centre pitch. Fig. 1 shows the main fabrication steps. In order to realize the $\mu \mathrm{LED}$ array with individually addressed n-electrodes, each $\mu$ LED element needs be fully isolated from both $\mathrm{p}$ - and $\mathrm{n}$ type $\mathrm{GaN}$ layers. To achieve this configuration, two steps of $\mathrm{Cl}_{2}$-based plasma etching are involved in the fabrication process. Firstly, GaN mesas are etched down to the sapphire substrate [Fig. 1(b)]. Then, a $\mu$ LED element is created at the centre of each mesa which stops at the n-type GaN [Fig. 1(c)]. Annealed Pd metal layer is used as a metal contact to p-type GaN. The metallization on the isolated n-type GaN mesa is realized by sputtering a Ti/Au metal bilayer. This bilayer is also patterned to make the metal track from the n-type GaN mesa so as to individually address each $\mu$ LED element through the n-electrode. After isolating each $\mu \mathrm{LED}$ element by a $\mathrm{SiO}_{2}$ layer, another Ti/Au metal bilayer is used to interconnect $\mu \mathrm{LED}$ elements forming a shared $\mathrm{p}$ electrode. Fig. 1(g) shows a schematic layout of the whole array to emphasise the common p-electrode and individually addressable n-electrodes. Compared with the conventional $\mu$ LED array design, the conductive paths are formed by Ti/Au metal bilayers rather than the $\mathrm{n}$-type $\mathrm{GaN}$ layer. Thus, series resistance differences between elements are much reduced owing to the significant lower sheet resistivity of the metal bilayer. 

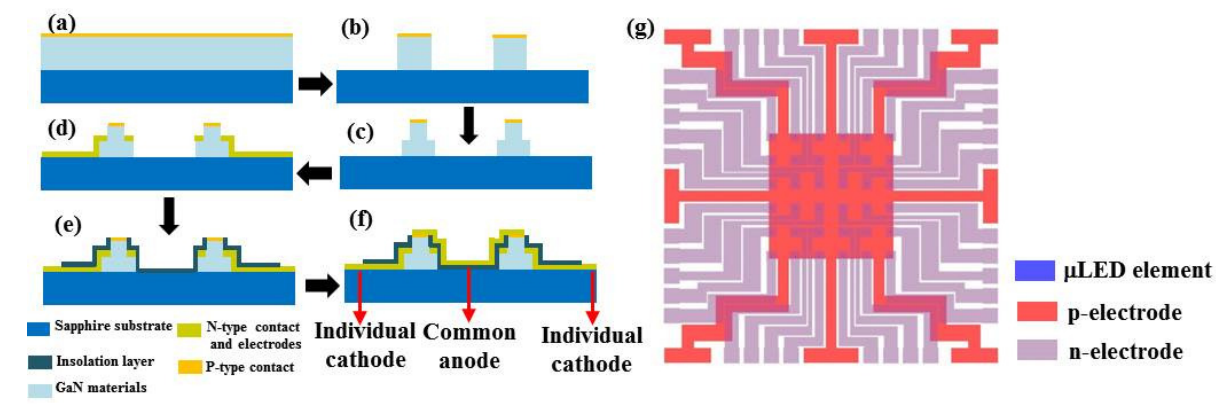

Figure 1: (a)-(f) Schematic diagrams of the fabrication process for the $\mu$ LED arrays. Part (g) shows a schematic layout of the whole $\mu$ LED array.

\section{PERFORMANCE AND APPLICATION OF $\mu$ LED ARRAYS}

Fig. 2(a) illustrates the current density-voltage and optical power density-current density characteristics of a single $\mu$ LED element in the array. This $\mu$ LED element can be operated at a direct-current current density up to $10.5 \mathrm{kA} / \mathrm{cm}^{2}$ and is able to produce a continuous wave optical power density over $414 \mathrm{~W} / \mathrm{cm}^{2}$. As mentioned, this high operating current density leads to a high modulation bandwidth as shown in Fig. 2(b). This $\mu$ LED element has an E-O modulation bandwidth in excess of $350 \mathrm{MHz}$, which is significantly higher than the value of typical commercial LEDs [3]. In order to illustrate the electrical/optical uniformity, the measured current and optical power densities at a fixed voltage of $7 \mathrm{~V}$ for 5 randomly selected $\mu$ LED elements are presented in Fig. 2(c). Analysis of the data reveals the variations of current and optical power densities are within $16.3 \%$ and $6.8 \%$, respectively. These variations are mainly caused by the different lengths of metal tracks connecting each $\mu$ LED element to its corresponding n-electrode and can be further reduced by increasing the thickness of metal tracks. The $\mu$ LED array is further integrated with the custom NMOS-based driver. Detailed information on this driver and integration process can be found in Ref.[2]. Fig. 2(d) shows the open eye-diagram obtained with two $\mu$ LED elements operating at $250 \mathrm{Mbps}$ under OOK data transmission scheme.
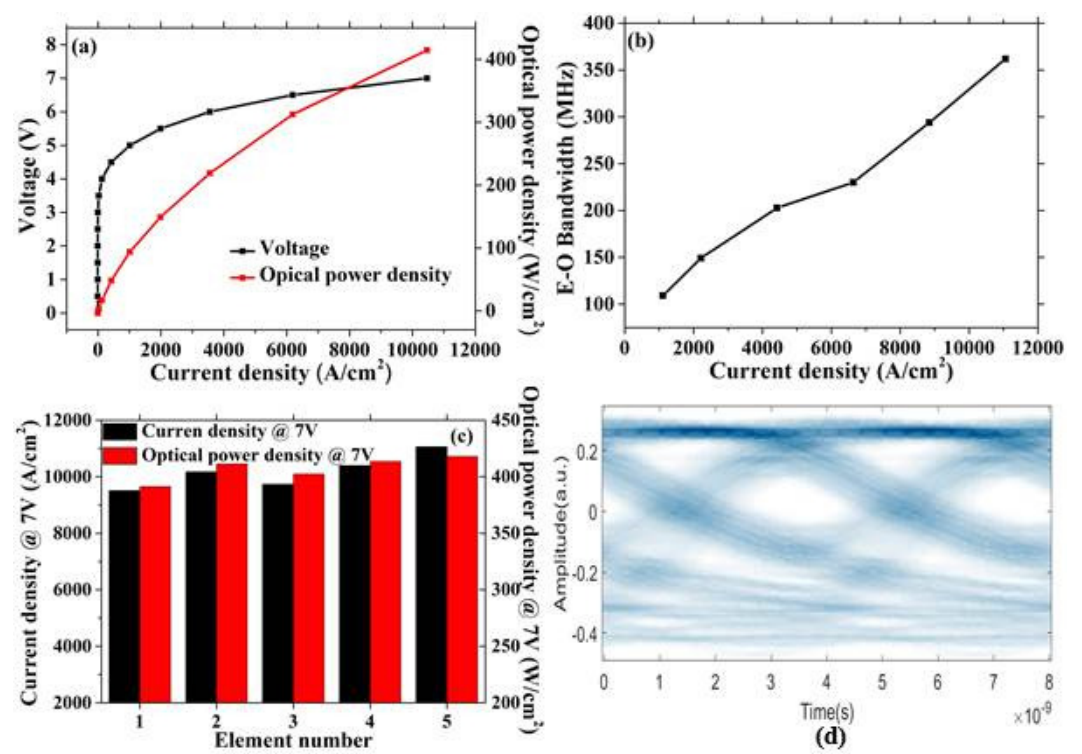

Figure 2: (a) Current density-voltage and optical power density-current density characteristics of a single $\mu$ LED element; (b) E-O modulation bandwidth characteristic of the same $\mu \mathrm{LED}$ element; (c) electrical and optical uniformities of 5 selected $\mu \mathrm{LED}$ elements in the array; (d) eye-diagram for two $\mu \mathrm{LED}$ elements operating at $250 \mathrm{Mbps}$ under OOK scheme by the NMOS-based driver.

\section{SUMMARY}

The fabrication, performance and application of the GaN-based $\mu$ LED array sharing a common p-electrode with individual-addressed $\mathrm{n}$-electrodes are demonstrated in this work. The novel configuration enables this array to be compatible with NMOS-based driver for faster modulation. The fabricated $\mu$ LED array shows promising performance. The application of this array integrated with an NMOS-based driver in VLC is also presented.

The work was funded by EPSRC grant EP/K00042X/1. Data is available online at http://dx.doi.org/10.15129/5dc8bacf-23f2-4e9a-afa3b7dd4fb7806a.

\section{REFERENCES}

1. Ferreira, R.X., et al., High bandwidth GaN-based micro$L E D$ s for multi-Gb/s visible light communications. IEEE

Photonics Technology Letters, 2016. 28(19): p. 2023-2026.

2. $\quad$ Rajbhandari, S., et al., High-Speed Integrated Visible Light Communication System: Device Constraints and Design

Considerations. IEEE Journal on Selected Areas in Communications, 2015. 33(9): p. 1750-1757.

Vučić, J., et al., $513 \mathrm{Mbit} / \mathrm{s}$ visible light communications link based on DMT-modulation of a white LED. Journal of Lightwave Technology, 2010. 28(24): p. 3512-3518. 